\title{
MÉTIERS ET FONCTIONS DE LA COMMUNICATION D'ORGANISATION : UNE MISE EN PERSPECTIVE GÉNÉRALE
}

\author{
Axel Gryspeerdt et Florence Carion ${ }^{1}$
}

Les sources d'information sur les métiers, les fonctions et les pratiques professionnelles de la communication d'organisation sont peu nombreuses : déjà en 1993-94, une étude avait montré les carences profondes de documentation sur les «métiers des relations publiques »: manque flagrant de récits professionnels, faible présence d'ouvrages vulgarisés et de notices pédagogiques, de même que d'œuvres de fiction ${ }^{2}$. Les sources les plus fiables étaient d'une certaine manière les moins disponibles, à savoir les profils de postes internes aux entreprises, en général confidentielles. Les offres d'emploi elles-mêmes beaucoup plus médiatisées ne semblaient pas, quant à elles, bénéficier, a priori, d'un taux de lecture élevé, à l'exception des moments liés à la recherche d'une situation. Un paradoxe semblait surgir de la confrontation entre deux données ; d'une part le grand intérêt, voire l'engoue-

1 Axel Gryspeerdt est professeur au Département de communication de l'Université catholique de Louvain. Florence Carion est assistante dans le même département.

2 Il s'agit d'une recherche dite «Tournesol», menée dans les années 1993-1994 en collaboration avec les enseignants des relations publiques à l'Université de Nancy II, sous la direction du Professeur Louis-Philippe Laprévote. Le relevé systématique de l'ensemble des documents existants à l'époque, dressé à cette occasion, n'a malheureusement jamais fait l'objet d'une publication.

Recherches en communication, $\mathrm{n}^{\circ} 25$ (2006). 
ment des jeunes pour les métiers de la communication d'organisation, ainsi que peut le laisser penser le nombre extrêmement élevé d'étudiants qui s'inscrivent dans les filières d'enseignement qui y préparent, et, d'autre part, la faible quantité de données publiquement disponibles dans leur environnement. L'attractivité des professions « des relations publiques » serait-elle corrélée à une méconnaissance du secteur, ou du moins à une faible information circulant à propos de celui-ci ? Comment les jeunes se sont-ils initiés à la professionnalisation du secteur ; dans quelle mesure la connaissent-ils et peuvent-ils la maîtriser ? En outre, on pouvait se demander si une faible médiatisation et publicisation des métiers et des pratiques de la communication d'organisation n'allaient pas engendrer des images stéréotypées, voire des «typifications » très restrictives selon des mécanismes de valorisation et de dévalorisation de certains aspects liés à ces métiers. Le contexte lui-même était-il porteur d'une « culture » implicite, plus ou moins typifiée rendant non nécessaires des explications davantage détaillées ? Cette « culture » donnaitelle des informations sur la nature des métiers, ou se contentait-elle de traiter du statut lié à ceux-ci ?

Plus récemment, dans le cadre d'un séminaire de second cycle ${ }^{1}$ ayant pour thématique les métiers de la communication d'organisation, les étudiants de l'Université catholique de Louvain ayant choisi la filière « relations publiques et communication d'organisation» ont été amenés à exprimer leur vision du secteur et leurs représentations des métiers. Dans l'ensemble, ils considèrent le domaine de la communication d'organisation comme dynamique et attrayant, mais relativement flou. Ils s'accordent sur des formules assez vagues, du genre « la communication organisationnelle fait penser au métier de chargé de relations publiques ou à divers métiers de la publicité ». Ces mêmes étudiants citent spontanément une grande diversité de métiers (plus d'une trentaine), mais parfois avec des occurrences très faibles, bénéficiant d'une bonne image générale, mais fortement méconnus de leur entourage. Les sources d'informations que les étudiants privilégient pour se renseigner sur ces métiers sont diverses : les cours dispensés, l'entourage, les centres de documentation, les associations professionnelles, les médias... L'information existe mais il n'est pas aisé, selon

1 Atelier de gestion d'image d'organisation (COMU 2281) du programme de second cycle en information et communication, Département de communication de l'UCL, année académique 2001-2002. 
eux, d'y accéder. A leurs yeux, un travail de centralisation de l'information sur les métiers est nécessaire. Ainsi, s'ils se disent prêts à consacrer quelques années d'études à s'y préparer, il leur est simultanément difficile d'exprimer avec quelque précision les fonctions liées à l'exercice professionnel, sans devoir passer par un lourd et fastidieux travail de recherche par exemple auprès des centres d'orientation professionnelle ou des services d'information sur les études et les professions, qui ne peuvent leur fournir que quelques renseignements. Il reste paradoxal de constater que les métiers attirent -sans doute par le statut qui est valorisé- alors qu'ils sont confondus les uns avec les autres et que les mots manquent pour les dénommer.

Les auteurs de cet article ont souhaité dès lors procéder à un examen de la situation plus actuelle et plus large dans le but de recouper les informations disponibles et de se pencher sur les dispositions inhérentes à ces métiers : nature du travail établi, délimitation avec d'autres professions relevant de secteurs plus ou moins proches -tels l'information, la communication, le commerce-, rôle joué par les professions relevant du secteur... Le but premier étant d'établir un relevé des professions sous forme d'un inventaire contextualisé dans la perspective de "révéler », en quelque sorte, la nature ou les principes de construction sociale de ces métiers.

C'est ce parcours que le présent article souhaite mettre en évidence, à partir, tout d'abord, d'un examen des sources habituellement disponibles; ensuite d'une recherche davantage pointue portant sur le contenu de quelques ouvrages parus dans la période allant de 1994 à 2006, débouchant sur un essai de typologie; enfin une tentative de compréhension du système que ces métiers ${ }^{1}$ mettent en place.

1 Nous reportant à la distinction, proposée par Pierre de Saint-Georges dans son article, entre phénomènes et politiques de communication, nous précisions que cet article s'attachera à éclairer les métiers liés aux stratégies et politiques de communication d'organisation. 


\section{Les représentations et les sources d'information sur les métiers}

Nous commencerons par aborder la question des représentations des métiers de la communication d'organisation et des sources d'information disponibles, à travers une présentation de la situation actuelle qui nous permettra d'évaluer globalement le niveau de connaissance des métiers.

\section{Un constat actuel}

A l'heure actuelle, le nombre de récits professionnels, sous forme d'interviews, de témoignages et de «mémoires » écrits par des praticiens des relations publiques et de la communication d'organisation semble très faible.

Alors que les milieux du journalisme et de la publicité s'expriment régulièrement dans des œuvres autobiographiques, des interviews ou des reportages vulgarisés dressant leur portrait, les communicateurs d'organisation apparaissent beaucoup plus discrets, sinon réservés. A quelques exceptions près, ils s'expriment très rarement sur leur travail. Ainsi, il n'existe pas dans ce domaine d'équivalent des récits autobiographiques d'un David Ogilvy, d'un Marcel Bleustein-Blanchet ou d'un Jacques Séguela ou encore d'un Jean-Claude Guillebaud, d'une Françoise Giroud, d'un Philippe Labro ou d'un Albert Londres. Même dans la sphère purement anglo-saxonne où quelques «mémoires » de grands professionnels ont été publiées sous forme de livres, ceuxci restent peu nombreux comparativement aux autres secteurs de la communication et ont rencontré un succès mitigé, restreint au milieu des connaisseurs, sans avoir été traduits en d'autres langues. Cette situation affecte non seulement leur notoriété propre -à peu près tous les étudiants qui se destinent à une filière d'enseignement en relations publiques ignorent le nom des 'fondateurs' de la discipline- mais aussi les représentations des métiers.

Cette discrétion peut s'expliquer de plusieurs façons. Les métiers de la communication d'organisation font partie des métiers de cadres d'organisation et ces derniers, à l'exception des cadres supérieurs patrons d'organisations, sont souvent peu enclins à s'exprimer sur eux- 
mêmes hors de la sphère purement professionnelle (par exemple dans des journaux ou bulletins d'entreprise). Ils sont d'ailleurs peu encouragés à le faire, vu qu'il n'existe guère de structures éditoriales, ni de lectorat et que les rares livres parus ont donné lieu à polémiques. Un des jeunes professionnels du lobbying s'est ainsi vu dénigré à la parution des révélations qu'il a pu faire sur ses activités, dans un ouvrage paru au début des années 1980. Les rares praticiens à s'être plus largement épanchés ont davantage parlé d'autres aspects de leur personnalité ou de leurs activités, ces derniers semblant davantage attractifs et impliquants que ceux relatifs à leur pratique des relations publiques ${ }^{1}$.

Si les professionnels semblent fort taiseux sur leurs pratiques, les médias ne sont pas plus enclins à les mettre en valeur, ni du côté document, ni du côté fiction.

Largement éclipsés par les top-managers, par les hommes politiques, par les vedettes du show business et par les journalistes, les communicateurs d'organisation apparaissent très peu dans les médias et lorsque des porte-parole ou des attachés de presse montent sur la scène publique, il s'agit moins de se montrer eux-mêmes en expliquant leurs pratiques professionnelles que de parler de leurs commanditaires et des actions de ces derniers. Les rares professionnels à être un peu plus 'vedettarisés' le sont essentiellement aux yeux de leurs pairs, lors de journées d'études et de colloques, et très rarement devant un public davantage composite. Ainsi, le grand public est-il en mal de pouvoir citer le nom de communicateurs d'entreprise, à quelques rares exceptions près ${ }^{2}$. Seuls semblent émerger les noms de communicateurs dont le parcours les a amenés à exercer préalablement des fonctions davantage publiques. Ainsi en estil, par exemple, de journalistes devenus communicateurs d'entreprise ou communicateurs politiques. Mais l'observation générale est confirmée

1 Ainsi, il est à relever qu'un des grands protagonistes belges des relations publiques par la pratique professionnelle, les conférences, l'action associative et l'enseignement, a, dans un livre d'entretiens commis avec un journaliste spécialisé en questions historiques, largement focalisé ses propos sur son rôle en tant que dirigeant de journaux, en tant qu'acteur de la vie intellectuelle et politique belge, et en tant que grand résistant pendant la seconde guerre mondiale, évoquant beaucoup moins sa carrière, pourtant longue, de relationniste.

2 Il s'agit de cas liés à la communication financière, ou à la communication de crise. Ainsi le porte-parole de l'Otan a-t-il acquis une certaine célébrité, dépassant le cercle des initiés. 
par le fait que la plupart des journalistes qui optent pour devenir communicateurs 'disparaissent' vite aux yeux de l'opinion publique.

Quant à la médiatisation fictionnelle, le tour en est vite effectué, dès lors que sont pointés quelques films de fiction, romans et extraits de bandes dessinées. Rien de comparable avec le nombre de héros de bandes dessinées ou de films qui exercent des fonctions de reporters, de photographes de presse, de rédacteurs ou de journalistes de tous types. Cependant, quelques films américains ont joué un rôle plus important dans la mise en scène de métiers liés au domaine. Sont à citer parmi eux, Le syndrome chinois (de James Bridge, 1979, avec Jane Fonda, Michael Douglas et Jack Lemon) qui fait la part belle au journalisme d'investigation et met en scène un chargé de relations publiques en exercice, et The american Président -en français Le Président et Miss Wade- (de Rob Reiner, 1995, avec Annette Bening et Martin Sheen) qui a modifié les perceptions du lobbying, en ayant comme héroïne principale une « spécialiste de la stratégie politique des organisations », défendant une cause écologique; ou plus récemment Thank you for Smoking (de Jason Reitman, 2005, avec Aaron Eckhart et Robert Duvall), où le porteparole d'un grand fabricant de tabac tente de rester un modèle pour sa famille. Du côté des séries télévisées, on peut relever Spin City (mettant en scène une équipe de professionnels s'efforçant de soigner l'image publique du maire de New York) ou A la Maison Blanche (présentant les coulisses de la Maison Blanche et l'équipe entourant le Président des Etats-Unis, notamment ses conseillers en communication et attachés de presse).

Alors que certains métiers donnent lieu à une vaste visibilité publique et sociale, permettant au grand public de s'en faire une représentation basée sur une ou plusieurs facettes des activités professionnelles -métiers et prestations professionnelles en relation directe avec le public- d'autres professions restent largement dans l'ombre, déployant leurs pratiques loin des regards, dans des bureaux ou des offices. L'ensemble des cadres d'entreprise, en outre souvent fort peu médiatisés, ainsi que cela a déjà été souligné, correspond aux métiers dont les prestations semblent les moins visibles. Les lieux où s'effectue le travail professionnel sont largement déterminants de ce peu de visibilité publique et sociale. 
Ainsi que l'un des auteurs l'a écrit dans un article consacré aux cadres politiques,

il suffit que certains lieux soient moins visibles par tous (seuls des cadres organisationnels, souvent appartenant au même groupe professionnel, peuvent les voir) et que certaines fonctions soient moins symbolisées, pour que la netteté professionnelle s'estompe et fasse place à l'ignorance et à la méconnaissance (que fait-il celui-là ; que fait-elle celle-là ?). Il en est ainsi de certaines professions à l'abri des regards (les membres ne sont pas vus dans leurs environnements de travail; ou ils sont vus sans que la nature de leur fonction ne soit perçue ou interprétée). Métiers sans visibilité et peut-être sans chances d'attrait hors de la sphère de ceux qui savent, ont été initiés ou instruits, ou sont liés par parenté ${ }^{1}$.

Les chargés de relations publiques et les directeurs de communication n'échappent guère à la règle de l'espace clos ou caché des cadres d'entreprise : la visibilité sociale donnée à leur prestation est faible. Le discours des attachés de presse et des porte-parole dont la fonction est davantage publique ou médiatisée, est, comme nous l'avons déjà évoqué, quant à lui moins centré sur les fonctions exercées par les agents professionnels que sur les activités et sur l'identité des entités dont ils sont censés parler: le mandataire s'estompe largement en faveur du commanditaire. Seule exception à la règle, déjà mentionnée, les anciens journalistes devenus communicateurs d'organisation font davantage parler d'eux et sont parfois mis sur la sellette, l'étanchéité du vaste système de communication étant interpellée par ce que certains appellent défection ou trahison.

Invisibilité des prestations, invisibilité des lieux, invisibilité aussi des objets; alors que le garagiste est qualifié par la dépanneuse, le garage et les outils de travail, le dentiste par la fraiseuse et par son cabinet, le facteur par sa mallette et son moyen de transport, ainsi que par le bureau de poste, aucun objet concret suffisamment préhensible par les publics ne semble symboliser la communication d'entreprise ${ }^{2}$.

1 A. GRYSPEERDT, «Communication sur les professions et renouvellement des cadres politiques », in Revue de l'Institut de sociologie, Bruxelles, Université libre de Bruxelles, $n^{\circ} 1-2,1995$, p. 68.

2 Sont exceptés ici les principaux outils des relations de presse, tels le communiqué de presse, la farde de presse, étant donné qu'ils sont en général destinés exclusivement 
Aux trois éléments évoqués ci-dessus s'ajoute une quasiabsence de travaux sociographiques ou monographiques décrivant les métiers.

Alors que dans le domaine des médias et même de la publicité abondent les centres d'études, les observatoires, les publications statistiques et descriptives sur les diverses fonctions, activités et pratiques professionnelles, seuls quelques guides pratiques de vulgarisation assez sommaires existent en ce qui concerne les métiers de la communication des organisations et des entreprises, donnant des renseignements de base sur les profils professionnels et les compétences demandées. Il est vrai qu'il n'y a pas non plus de centres d'études, ni d'observatoires de la communication d'organisation, alors que dans le domaine du journalisme et des médias, de la publicité et de la protection des consommateurs, il en existe de nombreux, qui n'hésitent pas à publier des monographies et des études statistiques. A quelques exceptions près (Italie, Hollande), les associations professionnelles n'ont pas davantage édité de livres ni publié de dossiers sur la question. Quant à l'enquête menée par Robert Tixier-Guichard et Daniel Chaize parue en 1993 sous l'intitulé «Les Dircoms »'1, elle s'avère être davantage un document de réflexion critique sur les stratégies suivies par les communicateurs, qu'une analyse socio-descriptive détaillée des fonctions que ceux-ci exercent dans le cadre de leur mandat; bien que le sous-titre évoque la perspective d'une enquête, le lecteur soucieux de trouver une approche sociologique de l'univers socio-professionnel des directeurs de communication, restera sur sa faim. A l'exception d'une importante thèse consacrée aux «avatars du modèle professionnel » que constitue le métier de directeur de communication ${ }^{2}$, très peu de travaux universitaires sont en outre consacrés à la question. Quant aux ouvrages écrits par des enseignants universitaires s'interrogeant sur les fonctions occupées dans le cadre de ces métiers, ils feront l'objet d'une analyse plus détaillée dans la suite de cet article.

à la catégorie des journalistes.

1 R. TIXIER-GUICHARD, D. CHAIZE, Les Dircoms. A quoi sert la communication? Enquête, Paris, Ed. du Seuil, 1993.

2 J. WALTER, Directeur de communication. Les avatars d'un modèle professionnel, Paris, L'Harmattan, 1995. 


\section{Un niveau de méconnaissance généralisée}

$\mathrm{Vu}$ cette absence réellement importante de 'sources' sur les métiers de la communication d'organisation et vu ce manque de visibilité professionnelle, on ne s'étonnera pas du niveau de méconnaissance en la matière, constatée auprès du grand public et aussi -phénomène tout aussi interpellant- auprès des jeunes qui se disent attirés par le secteur. Pour eux, comme pour leur environnement direct (parents, amis), le domaine reste quelque chose de flou, de difficile à cerner et à dénommer, de là leur avidité à rencontrer, lors de stages par exemple, des personnes qui pourront leur proposer des modèles professionnels concrets. Seule l'expérience directe semble être, ici, la source la plus féconde d'information; les autres sources potentielles n'étant guère alimentées, ainsi qu'en témoigne ce relevé. Les métiers du système de communication d'organisation sont très mal connus, fort peu identifiés et largement confondus. L'identité professionnelle semble, au premier abord, faible. La confusion semble régner, sauf aux yeux de ceux qui font partie du monde de la communication des organisations. Autrement dit, le nombre d'initiés dépasse à peine le nombre de pratiquants.

Sont congruents, en outre, avec ce constat de méconnaissance généralisée et de quasi absence de visibilité publique,

- le peu d'action informative proposée par les associations professionnelles, en dehors de l'information distribuée aux membres ;

- l'absence de lieux de rencontre organisés : il n'existe pas d'équivalent de maisons de la presse dans le secteur organisationnel ;

- l'inexistence de lieux clairement identifiés en tant que sources de renseignement sur le secteur de la communication d'organisation;

- l'absence d'un consensus sur les dénominations usuellement utilisées et de manière générale, le peu de 'relations publiques' sur les relations publiques, dans le sens large de cette expression, à l'exception de quelques dispositifs de promotion propres à susciter la méfiance.

Sur ce constat de méconnaissance généralisée des métiers de la communication d'organisation et de quasi-absence de 'relations publiques' des public relations officers, quelques éléments de réflexion peuvent prendre appui. 
C'est toute une partie du système d'information global de notre société contemporaine qui semble occultée. Alors que les dispositifs de communication médiatique sont souvent interrogés et mis sur le vif, le système de communication d'organisation, pourtant lié à la problématique dite des 'spin doctors', n'est guère l'objet de débats publics ou d'interrogation sociétale. L'opinion publique ne s'est par exemple pas encore rendue compte que le nombre de communicateurs d'organisation, tous métiers confondus, est devenu largement plus élevé que celui des journalistes, ce qui présente un certain nombre de conséquences de poids.

Alors que le journalisme fait l'objet de débats, la communication d'organisation ne semble donner que peu de prise à une réflexion généralisée, laissant les stéréotypes et les images toutes faites l'emporter. Fameux vecteur d'opinions et de représentations contemporaines, les blogs ne semblent pas modifier la donne. Alors que les managers, les hommes politiques, les journalistes, et toutes sortes d'opérateurs, même les plus anonymes, semblent s'exprimer sur le Web, rares sont les communicateurs d'entreprises à se saisir de ces nouvelles technologies pour donner une représentation de leur profession ${ }^{1}$.

Faut-il en déduire, comme l'hypothèse en a déjà été dressée par ailleurs ${ }^{2}$, un manque flagrant d'insertion de la communication d'organisation dans la vie culturelle, sociale et politique ? Ceux qui représentent les organisations ne jouent-ils pas un rôle capital dans ce domaine? Les faiseurs d'opinion ne méritent-ils pas que ceux qui véhiculent les opinions puissent davantage comprendre les enjeux et les défis professionnels qui les animent, ce qui exige une connaissance des tâches qu'ils sont concrètement amenés à remplir et un éclairage sur les fonctions qu'ils exercent ?

S'agit-il seulement d'un manque flagrant de vulgarisation ? Les personnes motivées peuvent-elles aisément trouver d'autres sources que celles évoquées dans le début de cet article ? Une abondance qualitative serait-elle susceptible de pallier à l'absence quantitative ?

1 Par contre, il existe des blogs d'informations sur les pratiques de gestion des ressources humaines, et bien entendu -voir suite de cet article concernant les tendances générales- des blogs de promotion d'asbl, de mouvements politiques ou d'entreprises.

2 A. GRYSPEERDT, «Pratique professionnelle des relations publiques d'entreprise et recherche en communication d'organisation : vers un nouvel interface ? », in Hermès, cognition, communication et politique, $\mathrm{n}^{\circ} 38$, Paris, C.N.R.S., 2004, pp. 148 à 154. 


\section{Un inventaire davantage nuancé}

Une démarche de recherche des auteurs a dès lors consisté à dépasser le simple relevé topographique des sources, en allant voir de plus près dans les bibliothèques et en menant des investigations davantage profondes dans le contenu de quelques ouvrages. Le manque de temps et de moyens les a néanmoins empêchés de combiner cette approche bibliographique avec une analyse plus ou moins pointue des offres d'emplois et des « jobs descriptions ». Si ces derniers présentent l'inconvénient de rester très souvent confidentiels -il s'agit de documents internes aux organisations recrutantes- et donc d'être difficiles sinon impossibles à récolter, il n'en va pas de même des offres d'emplois publiées sur les sites Web ou dans la presse. Les auteurs sont bien conscients de la capacité de celles-ci à fournir un matériau d'analyse intéressant, à condition de pouvoir les combiner à quelques facteurs culturels, tels la langue, la région, le pays... et ils se réservent la perspective de s'y atteler dans un avenir plus ou moins proche. Une recherche effectuée en France dans les années 1990 avait ainsi permis de constater que l'appellation « relations publiques » recouvrait des fonctions et des tâches variables selon les quelques pays examinés par l'enquête, parmi les pays adhérant à l'Union européenne.

\section{L'apport des ouvrages}

Que donne, dès lors, le passage en revue plus systématique des ouvrages concernant spécifiquement les métiers de la communication, trouvés dans les bibliothèques des instituts de formation universitaire?

La plupart des ouvrages ${ }^{1}$ recensés par les auteurs de cet article se contentent de fournir des descriptions sommaires de métiers vedettes, assez standardisées, voire stéréotypés ${ }^{2}$.

1 Il en est de même de quelques sites Web.

2 Les ouvrages consultés sont notammentB.HAQUIN, Lesmétiersdelacommunication, Paris, Le Monde, 1994, V. DURAND, Les métiers de la communication d'entreprise, Paris, PUF, 1999 et C. AUBREE, Les métiers de la communication, Paris, L'Etudiant, 2000. Les sites web consultés sont celui du CELSA, http://www.celsa.fr, Rubrique « Métiers et Carrières », et celui de SCIENCES COM, http://www.sciencescom.org, Rubrique « Le guide des métiers », consultés en mars 2002. 
En croisant ces différentes sources, il ressort que quatre métiers se retrouvent dans toutes les typologies, à savoir: le responsable de la communication interne, le directeur de communication, l'attaché de presse et le consultant. Ces différentes typologies présentent les métiers de manière plus ou moins détaillée, parfois fort sommairement, et y associent des fonctions, des tâches et des outils. Compétences nécessaires et diplômes à obtenir sont parfois mentionnés.

Rédigé par un universitaire, Jean-Luc Michel, un ouvrage non vulgarisé consacré aux métiers de la communication ${ }^{1}$ se détache du lot.

Cet auteur s'attache à rendre compte de la complexité et de la diversité des fonctions de la communication d'organisation, en présentant près de trois cents tâches de base, une trentaine de grandes fonctions (aussi appelées missions) et quinze métiers principaux. Cet ouvrage dépasse les descriptifs sommaires classiques des métiers et propose un panorama original et complet pour saisir la diversité des tâches et comprendre l'évolution dynamique des professions basée sur des recombinaisons incessantes de tâches de base. En effet, plutôt que d'aborder immédiatement les métiers, il a semblé plus judicieux à l'auteur d'étudier leurs constituants les plus déterminants. Cette inversion de la logique habituelle, qui consiste à commencer par les métiers puis à décrire les tâches afférentes, permet de rendre compte finement de la réalité et de la complexité de la communication, de manière systémique. Les métiers, s'ils sont soumis à de fortes évolutions, correspondent ainsi à des combinaisons de fonctions qui demeurent indispensables à la communication d'organisation.

L'auteur définit la fonction comme un ensemble de tâches élémentaires ou élaborées, reliées dans un but précis, pouvant faire l'objet d'un traitement isolé, d'une évaluation et se combiner avec d'autres fonctions pour aboutir comme par degrés à des ensembles de plus en plus complexes, allant jusqu'à des métiers ou des professions.

Jean-Luc Michel identifie ainsi, selon les combinatoires de tâches, de fonctions et de missions, quinze métiers « classiques $»^{2}$, huit métiers

1 J.-L. MICHEL, Les professions de la communication, fonctions et métiers, Paris, Ellipses, 1999.

2 Chargé de relations publiques, Attaché de presse, Métiers de la publicité, Chargé de la communication externe, Responsable de la communication externe, Chargé 
émergents ${ }^{1}$ et cinq métiers «du futur $»^{2}$. Le panorama fourni est dès lors suffisamment diversifié, évolutif et caractérisé par des implémentations variables dans l'espace-temps, révélant par cet aspect la dimension géo-historique des métiers des relations publiques et de la communication d'organisation. Sans viser à être exhaustif -l'absence, en tant que métiers, du lobbying, de la médiation, de certains aspects de la communication événementielle et de la communication culturelle notamment s'impose aux observateurs du domaine- Jean-Luc Michel donne toutefois une vision d'ensemble d'un secteur dans lequel les pratiques s'affinent en se spécialisant tout en permettant des combinaisons entre elles. Le grand nombre de tâches (plus de trois cents) rend compte de l'utilité assumée par les professionnels, œuvrant simultanément dans plusieurs champs.

L'observateur reste néanmoins quelque peu effrayé par la diversité des métiers énumérés par Jean-Luc Michel, approchant la trentaine. Un pareil nombre n'est-il pas susceptible d'occulter et de complexifier le domaine plus que le révéler? N'y aurait-il pas moyen de proposer une typologie davantage signifiante, car moins complexe et moins éclatée, ou des regroupements davantage significatifs, permettant de dégager en quelque sorte «l'âme » ou «la nature » des métiers, pris dans leur ensemble et selon quelques catégories distinctives, davantage discriminantes les unes des autres? Il semble en effet que dans la découpe opérée par Jean-Luc Michel, certains métiers se différentient des autres par les fonctions exercées (par exemple, le chargé de communication externe et le journaliste d'entreprise), tandis que d'autres correspondent plutôt à des différences de niveaux (opérationnel, fonctionnel, stratégique) et de parcours des personnes exerçant les pratiques profession-

de la communication interne, Responsable de la communication interne, Rédacteur en chef de magazines d'entreprise, Journaliste d'entreprise, Concepteur/réalisateur audiovisuel, Conseiller en stratégie de communication (interne ou externe), Directeur de la communication.

1 Concepteur de services interactifs, Technicien en PAO, Infographiste, Concepteur multimédia ou hypermédia, Responsable de communication globale, Conseiller en stratégie de communication globale, Chargé du mécénat ou du parrainage, Chargé de communication financière.

2 La formation, Le management interculturel, La communication internationale, La communication de réseau, La communication stratégique. 
nelles convoquées (par exemple, le chargé de la communication interne et le responsable de la communication interne).

\section{Un essai de typologie}

Sur base des questions qui précèdent, un brainstorming restreint a permis de proposer quelques classes principales de métiers à partir de l'identification de terrains professionnels paraissant davantage délimités. Certes, il s'agit ici d'un premier essai, n'excluant pas la possibilité d'élaborer d'autres typologies. Le rangement proposé s'opère autour de quelques grands territoires, jugés relativement séparés les uns des autres, ce qui n'exclut pas un certain métissage dans les pratiques concrètes.

La catégorisation ébauchée en cinq classes s'appuie sur le fait que des appellations synonymes sont souvent utilisées, permettant au sein d'une même catégorie des permutations entre métiers qui n'entravent pas la position différenciée que cette catégorie occupe par rapport aux autres catégories. Agir de cette façon permet d'éviter une dispersion extrême, tout en évitant une assimilation pure et simple de toutes les pratiques.

Les cinq catégories proposées à titre hypothétique, selon une règle de proximités intraclasses et de différences interclasses marquées, sont les suivantes :

- Généraliste en communication (RP)

- Spécialiste interface médias (AP)

- Editorialiste d'organisation (JE)

- ntermédiateur (OMB)

- Lobbyiste (LOB)

Sans entrer ici dans plus de nuances, les éléments suivants peuvent être rendus apparents :

Le généraliste en communication, quel que soit l'intitulé inscrit sur sa carte de visite, est un Responsable des Politiques (RP) d'information et de communication d'une organisation : directeur de communication, chargé de relations publiques, responsable de communication externe 
et/ou interne, attaché de relations publiques, responsable de communication événementielle, patron d'agence, consultant, etc ${ }^{1}$.

L'interface médias est responsable des relations avec les médias, par exemple attaché de presse (AP), porte-parole, directeur des relations avec les journalistes...

L'éditeur et le rédacteur d'organisation sont responsables des médias et des publications d'une organisation, y compris via les nouvelles technologies, ce qui élargit la définition de journaliste d'entreprise (JE).

L'intermédiateur organisationnel est en charge des relations, parfois conflictuelles, d'un ou de plusieurs services avec les utilisateurs : ombudsmen $(\mathrm{OMB})^{2}$, médiateurs, gestionnaires de conflits...

Le lobbyiste (LOB) est le plaideur des intérêts et des causes défendues par les organisations auprès des personnes ayant une compétence décisionnelle.

Ainsi s'élaborent quelques profils, qui ont paru suffisamment distincts que pour être énoncés dans le cadre de cet article. Cependant cette classification ne semble pas à l'abri de critiques potentielles. Trois d'entre elles ont particulièrement été pointées.

- D'abord, cette typologie exclut les intervenants du domaine de la communication d'organisation qu' elle juge extérieurs au système d'exercice professionnel en ou pour organisations, à savoir les chercheurs, les formateurs et les enseignants, les experts et divers intervenants en milieux organisationnels, dont la logique les apparente moins aux cadres d'organisation ou aux responsables exerçant dans des agences ou dans des bureaux de consultance. Cette exclusion permettra néanmoins de mieux saisir ce que les métiers retenus ont en commun ${ }^{3}$.

1 Des sous-classes ou sous-catégories peuvent être établies, selon la sphère de responsabilité : ex. communication interne, communication financière, communication de crise, etc. Voir aussi pages suivantes.

2 Etant donné qu' un bon nombre de professionnels belges, de langue française, comme de langue flamande, travaillant au sein de services de médiation ou de cellules de gestion des plaintes, se présentent comme «ombudsman», nous avons gardé ce terme, même s'il est d'étymologie étrangère (suédoise). Les puristes ne le déclinent jamais au féminin, ni au pluriel, sans doute parce que, bien souvent, il s'agit d'une fonction exercée collectivement.

3 Voir plus loin. 
- Ensuite, elle ne correspond pas à la découpe opérée par les organisations elles-mêmes au sein de leurs organigrammes. Ne s'y retrouvent pas les divisions opérationnelles rangées en grandes « directions », direction du marketing, donnant lieu à l'exercice des métiers de la publicité ${ }^{1}$; direction financière donnant lieu aux métiers de la communication financière; direction des ressources humaines donnant lieu à des pratiques professionnelles en matière de communication interne ; dimensions environnementales et écologiques, avec des pratiques de communication de crise et de communication de proximité ; préoccupations éthiques se traduisant dans des tâches de communication citoyennes ; domaine de hautes stratégies politiques donnant naissance à l'exercice des publics affairs et du lobbying ; services de relation avec les clientèles supervisant les fonctions d'intermédiation. Tout au plus peut-on y trouver parfois des éléments de similarité (ex. lobbyistes, (inter)médiateurs) ou de spécialisation : le communicateur financier n'est-il pas en quelque sorte le responsable de la politique d'information et de communication du secteur financier de l'organisation?

- Mais, il y a plus important. En donnant une image figée des métiers apparaissant les plus fréquemment dans les années 2000 à 2006, la classification proposée ne contredit-elle pas le principe selon lequel les métiers sont essentiellement évolutifs et dynamiques, et résultent de combinaisons toujours changeantes?

La prudence incitera à privilégier une voie tenant dès lors simultanément compte de la démarche combinatoire proposée par Jean-Luc Michel, avec le risque d'une diversité très élevée et d'un éclatement très grand, et de cette démarche classificatoire dont le risque consiste à figer des situations à un moment donné constituant seulement une étape dans la dynamique géo-temporelle des professions.

Enoncer ces modèles en cinq classes présente néanmoins l'avantage de pouvoir prélever, à un moment du déroulement selon l'espacetemps, des échantillons suffisamment discriminants pour opérer des

1 L'hypothèse d'une étanchéité entre la publicité et les relations publiques sera néanmoins posée dans la suite de cet article. 
analyses et des interprétations au sein de classes professionnelles, ni trop, ni trop peu différenciées les unes des autres ${ }^{1}$.

Il convient à cet égard de remarquer que la division proposée ici reste cohérente avec les classifications opérées par les professionnels eux-mêmes, au sein des associations chargées de les représenter. Le découpage en organisations professionnelles offre en effet une série de similarités : associations de généralistes en relations publiques, associations d'attachés de presse, associations de journalistes d'entreprise, associations de médiateurs, associations de lobbyistes; par ailleurs, les communicateurs financiers, vu leurs hautes spécialisation et qualification, se sont également regroupés dans des structures associatives. On retrouve à la base de ce constat, l'idée énoncée par Jacques Walter ${ }^{2}$, selon laquelle ce sont ceux qui exercent les métiers eux-mêmes qui « créent » les professions.

\section{L'émergence d'un monde de mandataires}

Nous devons à présent dépasser le niveau de la typologie proposée pour tenter de comprendre le monde et le système complexes au sein desquels évoluent ces métiers de la communication d'organisation et qu'ils contribuent à façonner.

Ceux qui pratiquent ces métiers exercent leur compétence soit, dans une structure intégrée au sein d'une organisation, de type cellule, équipe, service, département, direction, en fonction du nombre de personnes occupées; soit, dans une structure externalisée ; soit, encore, dans un bureau ou une agence-conseil, totalement extérieure, fournissant des prestations selon un régime de commandite. Il est possible d'inclure sous cette catégorie le conseiller indépendant ou le consultant travaillant seul, ayant éventuellement un autre emploi principal (ex. l'enseignant-consultant), mais pas le formateur, ni l'enseignant, ni encore le chercheur universitaire. Les professionnels du domaine peuvent aussi exercer leur pratique selon trois principaux niveaux ; un niveau straté-

1 La mise en perspective des métiers, demandée à Axel Gryspeerdt, avec le concours de Florence Carion, avait notamment pour but de permettre aux membres du séminaire de recherche dont ce numéro de la Revue est issu, de sélectionner des « groupes » de professionnels à rencontrer, afin de «débattre » au sein de chacun des groupes ainsi constitués.

2 J. WALTER, Op. cit. 
gique (ex. élaboration de plans stratégiques, de programmes de communication, de dispositifs nouveaux...), un niveau opérationnel (exécution et application) et un niveau purement logistique (d'appui technique).

Qu'ont donc en commun cet ensemble de métiers, variant de cinq principales classes à plus d'une trentaine, selon l'angle choisi ?

\section{Un monde de la communication des organisations}

Une première manière de les soumettre à l'examen consiste à se rendre compte que, quel que soit leur niveau de pratique et leur lieu d'exercice, ils peuvent tous, plus ou moins, relever du management ${ }^{1}$. Ainsi, un spécialiste des sciences de la gestion pourra-t-il sans difficulté les faire figurer parmi les professions de cadres d'entreprise, dont il a pu être observé qu'ils passent une partie considérable de leur temps à des fonctions de communication ${ }^{2}$.

Mais si une découpe en tant que cadres supérieurs ou moyens d'entreprise peut aisément se justifier, un rattachement aux métiers de la communication et de l'information est tout autant envisageable. Sans que cela ne soit exclusif, le domaine d'exercice professionnel est en effet un critère de rattachement, qui peut sembler rempli de sens et de significations. Selon cette perspective, les métiers en question relèveraient du secteur de la communication et constitueraient au sein de ce secteur, sans doute très vaste, un système propre. Une thèse récente énoncée par Hervé Collet conforte ce choix. Ainsi, dans son ouvrage consacré à la communication sociale, publié en 2004, Hervé Collet rappelle que les acteurs sociaux de l'après-seconde guerre mondiale se sont accordés pour distinguer dans le monde occidental trois principaux systèmes de communication autonomes, à savoir le journalisme, la publicité et les relations publiques. Il donne à ces trois systèmes le nom de «disciplines glorieuses ou de disciplines reines ». Il ajoute que les relations publiques se sont 'dissoutes' dans la communication d'organisation ${ }^{3}$. Au cours des cinquante dernières années, ce tripode disciplinaire, qui s'appuie sur un réseau de règles déontologiques ou juridiques ${ }^{4}$, s'est

1 C'est un peu moins vrai des métiers de la logistique.

2 H. MINTZBERG, Le manager au quotidien, Paris, Ed. d'organisation, 1984.

3 H. COLLET, Communiquer, pourquoi, comment? Le guide de la communication sociale, Eaubonne, Cridec éditions, 2004, p. 54.

4 En France, l'arrêté du 23 octobre 1964 du Ministère de l'Intérieur spécifie que 
retrouvé amplifié par un ensemble de pratiques constituées en domaines professionnels et en filières d'enseignement quasi-totalement étanches, selon Hervé Collet.

Un ensemble de composants divers constitue le fondement et la culture propre à chacun des trois principaux systèmes de communication. Ces derniers sont en effet constitués de méthodes de travail, de langages spécifiques, d'ensembles de règles, de structures professionnelles, de dénominations, de dispositifs d'action et de séries d'acteurs ${ }^{1}$.

Trois grandes étapes antérieures à la situation de l'après-guerre sont évoquées par lui, montrant par là que ce tripode est le fruit d'une évolution historique. Depuis lors, souligne-t-il, de nouvelles «disciplines assimilées » tendent à s'en dégager et à se constituer en objets d'organisation professionnelle et d'enseignement. Parmi ces disciplines assimilées, il cite le parrainage-mécénat, le lobbying, la communication interne et la communication des grandes causes sociales.

Malgré le fait que les trois grands systèmes de communication reposent sur un accord social, pratiqué et largement institutionnalisé, les éléments qui composent ces systèmes sont davantage flous : aucun vaste compromis social n'entérine en effet ni les dénominations des pratiques professionnelles -fortement localisées selon les pays, selon les organisations et selon les locuteurs- ni les relations, que ce soit au sein du système de communication, avec les autres systèmes ou avec les systèmes extérieurs à la communication, ni encore les délimitations internes au système.

Autrement dit, il n'existe dans ce secteur ni de corps totalement institué de métiers, ni de désignation labellisée ou certifiée par une quelconque instance. Ainsi, selon les lieux et les énonciateurs, porte-parole, attachés de presse, chargés de relations publiques et responsables de communication externe peuvent être tour à tour des synonymes ou bien des dénominations qui renvoient à des métiers très différents. Les termes ne sont pas protégés et sont fort fluctuants : un même terme pouvant

«Art.3. Les activités de conseiller en relations publiques et d'attaché de presse sont incompatibles avec celles de journaliste professionnel et d'agent de publicité » (cité dans H. COLLET, Op. cit., p. 52).

1 Ibidem, p. 49. 
désigner, selon les usages culturels, des pratiques fort différentes ${ }^{1}$. Ce constat vient entériner le fait que, dans le système de communication relations publiques-communication d'organisation, les identités professionnelles sont relativement complexes. Des divergences significatives apparaissent : alors qu'il est relativement facile de comprendre que les fonctions principales d'un expert-géomètre consistent à mesurer et à estimer la valeur d'un bien, les fonctions d'un communicateur d'organisation sont largement plus floues. S'intéressant au modèle professionnel du directeur de communication, Jacques Walter n'allait-il pas jusqu'à écrire que « la communication est décidément une histoire de flou $»^{2}$ ?

En empruntant à Howard S. Becker l'idée de « monde », se dégage une approche complémentaire selon laquelle le système de communication d'organisation aurait fait émerger un monde propre, avec l'aide de toute une série de collaborations.

Dans son ouvrage consacré aux mondes de l'art ${ }^{3}$, Howard S. Becker met en effet en évidence qu'un monde se construit à partir de collaborations multiples de tout un ensemble d'agents sociaux en activité de travail. Ainsi, le monde de l'art n'est pas construit (seulement) par les artistes, mais par un large ensemble d'intervenants qui l'aident à se constituer : critiques, conservateurs, galeristes, amis des artistes, exposants, responsables d'institutions muséales, gardiens de parking, préposés à l'accueil, architectes de musées, éditeurs de catalogues, imprimeurs de reproductions artistiques, journalistes spécialisés, etc. Il en est de même du monde du tramway ${ }^{4}$ ou de l'automobile, construits par un large ensemble de contributeurs institutionnels : inventeurs, entrepreneurs, producteurs, diffuseurs, utilisateurs, prêteurs, etc. Comme le signale Catherine Bertho Lavenir ${ }^{5}$ dans un ouvrage consacré au tourisme, «toutes sortes d'institutions se sont interposées entre le voyageur et sa passion » : entreprises éditoriales et bulletins associatifs,

1 En ce sens, une analyse des champs lexicaux pourrait être intéressante, à condition d'en corréler les résultats avec les contextes culturels. Il s'agirait, par exemple, d'établir une liste référencée à partir d'un relevé systématique des titres sur les cartes de visite ou dans les offres d'emplois.

2 J. WALTER, Op. cit., p. 187.

3 H. S. BECKER, Les mondes de l'art, Paris, Flammarion, 1988.

4 D. BOORSTIN, Histoire des Américains, Paris, Armand Colin, 1981.

5 C. BERTHO LAVENIR, La roue et le stylo. Comment nous sommes devenus touristes, Paris, Ed. Odile Jacob, 1999, p. 10. 
compagnies de chemins de fer, tour operators, systèmes d'hôtellerie et de restauration, parcs de loisirs, imageries publicitaires, sociétés de constructions d'aéroports, de routes et de parkings... En dehors du jeu des multiples acteurs, le monde en question n'existerait pas.

Dès lors, sans professionnels pour le pratiquer, sans enseignants pour y former, sans managers pour y croire et recruter ou mandater, sans utilisateurs, sans associations, sans imprimeurs, graphistes ou journalistes, etc., il n'y aurait pas de monde -ou de système- de communication d'organisation dans la société occidentale contemporaine. Car il s'agit-là, de même que dans les autres secteurs de la vie contemporaine, d'un phénomène historique, progressif et évolutif, qui aurait pu ne pas exister et qui, en ce qui le concerne, est corrélatif du développement fabuleux pris par les organisations elles-mêmes et de l'augmentation de la curiosité publique et médiatique pour les faits qui les caractérisent.

Non seulement le monde ainsi constitué est distinct des deux autres «mondes » de la communication (publicité et journalisme), avec lesquels il peut collaborer, et vis-à-vis desquels il constitue un système relativement imperméable, mais il est aussi autonome des autres systèmes, y compris des systèmes artistiques, éducatifs, documentaires et éditoriaux, dont la composante communicationnelle est loin d'être négligeable.

\section{Des mandataires spécifiques}

Une seconde manière d'envisager les points communs existant entre les divers métiers relevés, consiste à considérer que chacun de ces métiers dépend financièrement d'une entité payante ou, plus exactement, que les prestations effectuées par les agents en question sont rémunérées par un ou des commanditaires, dont ils dépendent directement (salaires) ou indirectement (honoraires, indemnités). Ces agents auraient ainsi reçu un mandat portant sur des matières relevant de la communication de la part d'un ou de plusieurs mandant(s), en général organisationnels ${ }^{1}$.

1 J. GIRIN, «Le langage et la compétence des agencements organisationnels », in Connexions, nº5, Paris, Erès, 1995, pp. 121 à 141. 
Il est dès lors possible de définir comme 'professionnels' de ce monde tous ceux qui œuvrent sous la houlette d'un mandant dans le cadre d'une mission portant sur une question de communication au sens large et ceci, quels que soient leur formation ou le diplôme obtenu, et quels que soient leur structure de travail ou leur niveau d'élaboration des tâches.

En fonction de la période, de la région et de la nature organisationnelle -hôpitaux, administrations, grandes entreprises, PME, milieux politiques, universités-, le contenu des mandats peut plus ou moins varier, les commanditaires et les mandataires n'ayant pas nécessairement suivi les mêmes parcours. D'une même façon, chaque organisation spécifique peut 'entrer' en communication et faire appel à des professionnels du domaine à des moments différents de son histoire et selon des opportunités ou des modalités différentes (communication événementielle, système d'identité visuelle, communication de crise, lobbying, etc.) ${ }^{1}$. Vu la nature historique de la construction du domaine, la variable géo-temporelle n'est en effet pas négligeable : le système de communication d'organisation est en perpétuelle transformation et évolution.

Le mandat contractualisé place les agents mandataires dans une situation de personnes recrutées, engagées et/ou financées en fonction de leur utilité organisationnelle : dans la toute grande majorité des cas, leur part d'autonomie et leur part de jeu personnel sont réduites au profit de l'entité collective dont ils dépendent. Quelques très rares exceptions peuvent exister de personnes payées par des tiers, qui seraient eux-mêmes indépendants, ou de personnes dont la liberté d'agir, de communiquer et d'informer serait garantie par une charte, un code ou des clauses particulières ${ }^{2}$.

1 J.-L. Michel précise à ce propos que l'on observe encore deux «marchés », un «marché de conquête (création de postes de communication dans les PME, administrations...) » et un « marché de renouvellement » (services de communication embauchant des diplômés pour des tâches précises de plus en plus diversifiées).

2 Il est à signaler à cet égard que les journalistes sont également rattachés à une organisation dont ils constituent une des ressources humaines, ou avec laquelle ils travaillent selon des contrats de free lance. Toutefois, en ce qui les concerne, existe un attirail plus ou moins développé de garanties statutaires, allant de la clause de conscience à la liberté rédactionnelle la plus large. 
Par des règles juridiques, par des sanctions venant de l'opinion publique et par quelques règles de fonctionnement organisationnel implicites au secteur (ordres de journalistes, rôle régulateur des instances associatives professionnelles ou contrôle des médias), les métiers des autres systèmes de communication paraissent davantage contrôlés par exemple, les journalistes porteurs d'actions d'entreprises cotées en bourse sont parfois tenus à déclarer leur statut d'actionnaire et à faire état de leurs possessions- ou appuyés dans leurs efforts d'autonomisation.

Le souci de l'opinion publique et des législateurs ne serait-il dès lors pas davantage élevé, là où les métiers reçoivent une visibilité sociale et médiatique plus grande ? Le fait que le résultat du travail professionnel des publicitaires et des journalistes s'effectue sous les yeux des consommateurs et des citoyens, au sein de vitrines médiatiques, n'aurait-il pas sinon provoqué, du moins accéléré la création d'un cadre plus exigeant en matière de prestations professionnelles ?

Selon le point de vue des sciences de la gestion, l'ensemble des pratiques professionnelles visées peut d'ailleurs s'envisager sur base de leur dimension de management. Ainsi, appliquant les idées d'Henry Mintzberg ${ }^{1}$, il ne serait pas impossible de considérer l'ensemble de ces métiers selon un point de vue managérial. Mais si les métiers et les activités en question relèvent du management et de l'expertise, ils peuvent être considérés également à partir de la spécialisation exercée, et donc de la communication vu que le mandat qui leur est confié porte sur des opérations de type «information et communication».

\section{Une hypothèse d'opacité professionnelle}

En guise de conclusion, nous pouvons nous demander dans quelle mesure les relatives, mais fort présentes, opacité, méconnaissance et peu de visibilité des métiers de la communication d'organisation ne sont-elles pas productrices simultanément d'une illusion de protection, se manifestant par une certaine discrétion et un certain secret sur le métier, en en réservant l'éclairage aux seuls initiés ${ }^{2}$; et d'un impératif

1 H. MINTZBERG, Op. cit.

2 Ce qui ne se traduirait pas, chez les acteurs du secteur, par un manque de sentiment d'être «professionnels de la communication d'organisation »; au contraire, selon 
professionnel consistant à braquer l'éclairage sur les commanditaires et leurs actions, en créant de manière dérivée, une opacité sur les principaux acteurs de la fabrication des images?

L'éclairage des organisations est en effet le résultat d'une série d'opérations de médiations et d'interpositions entre l'organisation et ceux qui sont en contact avec elle ; en aucun cas, il ne s'agit d'un processus spontané, naturel ou direct. Eclairer est un acte volontaire consistant à introduire des médiations pour faire ressortir l'un ou l'autre aspect. L'entreprise et l'organisation ne sont pas, en elles-mêmes, directement accessibles. Leur transparence et leur « immédiateté » sont illusoires. Ce qui est montré d'elles est le résultat d'un travail de médiations successives.

Autrement dit, dans le domaine de la communication des organisations, tout se passe comme si des médiations cumulatives s'étaient succédées. Sans que ce modèle n'ait été validé, il n'est pas impossible de faire ici l'hypothèse que les premières médiations aient été d'ordre commercial (la vente, la réclame, la publicité), informationnel, technique, relationnel, politique (affaires publiques), puis environnemental, éthique (citoyenneté et responsabilité sociale d'entreprise), etc., l'organisation étant montrée à la fois au travers de filtres (perceptions, images, opinions, jugements), de dispositifs (canaux, supports,...) et d'objets (symboles, bâtiments,...). Il est ainsi souvent relevé par des observateurs ou par des témoins qu'avant 1970 la grande majorité des organisations s'occupaient moins (sinon pas du tout) de communiquer avec des publics, mais principalement de produire, d'agir et de travailler. En cela, les premières médiations ont été d'ordre commercial : s'intéresser aux clients et s'interposer entre les consommateurs et les produits, afin de se positionner et se distinguer des concurrents, par le biais d'objets promotionnels et publicitaires, d'institutions (la réclame, le marketing, la publicité) et de personnes : les publicitaires, les photographes d'entreprise, les délégués commerciaux, les représentants de commerce, les chargés de relations extérieures... Ensuite, la logique informationnelle prime (en interne, principalement). Puis, les médiations se sont faites davantage relationnelles (construction d'une relation avec le consom-

J.-L. Michel, on assisterait à « un étrange phénomène dans lequel l'identité du communicant est d'autant plus forte que sa définition est floue» (J.-L. MICHEL, Op. cit., p. 10). 
mateur ou gestion relationnelle des ressources humaines) et politiques (communication avec les stakeholders et lobbying). Plus récemment, les consommateurs s'intéressant de plus en plus aux contributions de l'organisation en terme social, d'environnement et de développement, les professionnels s'entretiennent sur ces domaines avec les publics internes et externes.

En quelque sorte, les communicateurs d'organisations s'interposent entre l'objet de leur éclairage -les organisations et leurs composantes- et les collectivités humaines. Le mandat reçu par eux consiste à diffuser, transporter et modifier les représentations qui sont liées à cet objet. Mais, il convient de relever qu'ils ne sont pas les seuls acteurs à vouloir agir sur les représentations. Les acteurs des deux autres grands systèmes de communication, qu'ils soient journalistes ou publicitaires exercent également un rôle en matière de façonnage des représentations collectives et de création et de propagation d'image.

Ce processus de création d'image des organisations est simultanément un processus de constitution des publics. Sans le travail collectif sur les représentations, il n'est guère de publics, la reconnaissance des publics étant le résultat attendu de l'action menée par des professionnels aux intérêts complémentaires et parfois divergents ${ }^{1}$.

Ainsi, les communicateurs d'organisation apparaissent-ils davantage comme des éclaireurs et des médiateurs, chargés de mettre en lumière les organisations qui les commanditent, plutôt que des «monstrateurs » d'eux-mêmes. En suivant cette manière de raisonner, il est possible de se demander si la dose d'opacité remarquée n'est pas consubstantielle à l'exercice de leur profession ; si elle ne révèle pas profondément leur travail.

Lever le voile sur les composants de leur métier -ce dont il a été largement relevé qu'ils étaient loin de le faire eux-mêmes- et sur ce que ces métiers signifient en terme d'exercice professionnel (combien sontils ? où les trouve-t-on ? où laissent-ils leurs témoignages ?), permettrait dès lors une mise en visibilité susceptible de les révéler auprès de

1 Communicateurs d'entreprise, journalistes et publicitaires sont ici simultanément visés. Les publics ne perçoivent les organisations que par le filtre de leurs actions de médiations, d'éclairage et de traduction. 
l'ensemble des agents sociaux et d'éclairer leurs pratiques pour une plus grande lucidité sociale ${ }^{1}$. Ainsi se déploierait un processus d'éclairage sur les métiers eux-mêmes.

1 Un « Observatoire des métiers de la communication», menant des recherches sur la professionnalisation des métiers de la communication d'organisation, tel que pensé actuellement par Patrice de la Broise permettrait de rencontrer cette nécessité. 